\title{
Ensino do Pensamento Computacional por meio de Internet das Coisas: Possibilidades e Desafios
}

\author{
Gelson André Schneider ${ }^{1}$, Flavia Bernardini ${ }^{1}$, Clodis Boscarioli ${ }^{2}$ \\ ${ }^{1}$ Instituto de Computação/Universidade Federal Fluminense \\ Av. Gal. Milton Tavares de Souza, s/n - São Domingos, Niterói/RJ - CEP: 24210-310 \\ ${ }^{2}$ PPGComp/Universidade Estadual do Oeste do Paraná \\ Rua Universitária, 1619 - Faculdade, Cascavel/PR - CEP: 85819-110 \\ gelson.schneider@ifto.edu.br, ffcbernardini, boscarioli\}@gmail.com
}

\begin{abstract}
The educational processes is to develop in students the criticality and the ability to solve problems. Computational Thinking is being used for this, it can be accomplished through robotics, game design or unplugged computing. On the other hand, technologies for implementation Internet of Things are widely used in various sectors of society, and can be used for teaching Computational Thinking is a real possibility. We present in this study a Systematic Literature Review to analyze how Internet of Things is used for the teaching of Computational Thinking and we analyze important issues in this context.
\end{abstract}

Resumo. Processos educacionais têm como um de seus objetivos desenvolver nos estudantes a criticidade e a capacidade de resolver problemas. O Pensamento Computacional tem sido usado para isso, aliado ao uso da robótica, construção de jogos ou computação desplugada. Por outro lado, tecnologias para implementação de Internet das Coisas têm sido amplamente utilizada em diversos domínios da sociedade, e pode ser usada para ensino do Pensamento Computacional. Apresentamos neste trabalho uma Revisão Sistemática de Literatura para analisar como a Internet das Coisas é utilizada para o ensino do Pensamento Computacional, e analisamos questões importantes nesse contexto.

\section{Introdução}

A Tecnologia da Informação está inserida no cotidiano da maioria das pessoas. No entanto, para [Blikstein 2008] o uso da tecnologia não se limita a saber navegar na internet, enviar e-mail, publicar um blog, ou operar um processador de texto. Há os aspectos de mudanças sociais, de processos e de inovação, pelos quais diversas áreas obtiveram contribuições significativas pelas tecnologias. Na Educação, por exemplo, várias soluções digitais surgiram, sejam para auxílio nos processos de gestão ou para uso pedagógico.

A capacidade de usar os princípios da Ciência da Computação para desenvolver competências e habilidades para a análise e resolução de problemas chamamos de Pensamento Computacional (PC). Para [Wing 2006] o conceito fundamental do PC é a resolução de problemas por meio da abstração de um modelo computacional e, segundo [Blikstein 2008], por meio do PC é possível desenvolver a "habilidade de transformar teorias e hipóteses em modelos e programas de computador, executá-los, depurá-los, e utilizá-los para redesenhar processos produtivos, realizar pesquisas científicas ou mesmo, otimizar rotinas pessoais". 
VIII Congresso Brasileiro de Informática na Educação (CBIE 2019)

Anais do XXX Simpósio Brasileiro de Informática na Educação (SBIE 2019)

Coisas inteligentes conectadas à internet que podem captar, processar e transmitir informação é uma realidade cada vez mais presente em nosso dia-a-dia, esse ecossistema é conhecido como Internet das Coisas (do inglês, Internet of Thinks, IoT). Esta tecnologia tem impacto em todos seguimentos da sociedade, como saúde, educação, segurança pública e mobilidade urbana. Compreender as possibilidades, limitações e desafios do uso da IoT é uma habilidade importante para estudantes do século XXI.

O intuito da IoT no ensino do PC é desenvolver habilidades e competências no estudante que, além de aprender conceitos de Ciência da Computação, podem despertar seu interesse em criar soluções de problemas reais do seu cotidiano como hortas, casas e cidades inteligentes, tornando-o capaz de compreender e agir na sua realidade local, bem como fomentar o seu interesse em seguir carreiras relacionadas a essa área.

É nesse contexto que emerge essa pesquisa, que traz uma Revisão Sistemática da Literatura (RSL) acerca de como é realizado o ensino do PC por meio dos conceitos de IoT, bem como levantar questões a serem consideradas nesse contexto. Convém salientar que o foco não é o uso da IoT, de seus objetos e ferramentas para o ensino de computação [e/ou outros conteúdos], mas como a IoT pode ser útil para o ensino de PC.

Este trabalho segue assim organizado: na Seção 2 apresentamos o referencial teórico do trabalho, sobre conceitos relacionados ao PC e a IoT, de forma a relacionálos para o desenvolvimento das habilidades de abstração e raciocínio lógico; a Seção 3 descreve a metodologia empregada para execução da RSL; a Seção 4 traz as análises dos trabalhos selecionados, buscando responder às questões de pesquisa; a Seção 5 detalha uma análise do cenário atual e as perspectivas do uso de IoT para ensino do PC; por fim, as considerações finais estão na Seção 6.

\section{Referencial Teórico}

A ideia do desenvolvimento do PC vem sendo explorada desde os anos 50, embora não fosse cunhada com esse termo, a exemplo do trabalho de [Papert 1980], que diz que a apropriação das ferramentas tecnológicas por parte das crianças fornece um arcabouço rico para que sejam capazes de criar novos conhecimentos por meio de resolução de problemas utilizando conceitos da Computação. O termo Pensamento Computacional (Computational Thinking) foi apresentado apenas em 2006 por Wing [Wing 2006]. Segundo a autora, PC é a capacidade de utilizar os conceitos fundamentais da Ciência da Computação para resolução de problemas nas mais diversas áreas do conhecimento.

O PC auxilia na formulação de problemas e soluções representadas de forma que possam ser executados por processadores de informações computacionais ou humanos, melhor ainda, por uma combinação de ambos. Para Wing [Wing 2006] o PC pode ser organizado em quatro etapas: (i) Decomposição; (ii) Padrões; (iii) Abstração e (iv) Algoritmo. Pensar computacionalmente pode tornar o aprendizado mais significativo e motivador [Blikstein 2008].

Existem iniciativas para inclusão do PC como ciência básica em vários países. No Brasil, apesar do PC não fazer parte do currículo escolar, estudos mostram o crescente interesse dos pesquisadores sobre o tema. Em [França and Tedesco 2015a] são elencados os desafios e oportunidades ao ensino do PC na Educação Básica. Já Ortiz e Pereira [Ortiz and Pereira 2018] fizeram um mapeamento sistemático sobre as iniciativas 
VIII Congresso Brasileiro de Informática na Educação (CBIE 2019)

Anais do XXX Simpósio Brasileiro de Informática na Educação (SBIE 2019)

para promover o PC. Para esses autores "no Brasil, embora os primeiros resultados mapeados datem de 2015, indicando um estágio inicial de pesquisas no tema, já é possível observar uma expressiva quantidade de publicações, demonstrando avanços na área”.

Diferentes estratégias estão sendo pesquisadas para promover o ensino do PC, tais como: a Computação Desplugada: as atividades são realizadas sem o auxílio de tecnologias computacionais. No trabalho de [Werlich et al. 2018] foi realizada uma atividade com estudantes de duas turmas do $1^{\circ}$ e $2^{\circ}$ ano do Ensino Fundamental I. Na atividade os estudantes, com auxílio de um mapa, deveriam localizar o tesouro optando pelo caminho que utilizasse a quantidade mínima de retângulos, referência a um clássico problema em Ciência da Computação denominado de problema do caminho mínimo. Outra estratégia é o desenvolvimento de objetos de aprendizagem: [Kaminski and Boscarioli 2018] apresentam um roteiro de integração para o desenvolvimento do PC por meio da programação visual com o Scratch ao conteúdo curricular de estudantes do Ensino Fundamental I de uma escola pública. Os estudantes são desafiados a produzirem objetos de aprendizagem que são compartilhados com outros estudantes e, ainda segundo o estudo, foi possível constatar as contribuições do uso do Scratch para o desenvolvimento cognitivo por meio do PC.

Além da computação desplugada e do desenvolvimento de objetos de aprendizagem, são pesquisados o ensino de matemática, o desenvolvimento de jogos e a utilização da robótica. Em [Barcelos et al. 2015] é apresentada uma Revisão Sistemática da Literatura sobre as relações entre PC à matemática. Segundo os autores, os estudos procuram desenvolver conjuntamente o PC a Matemática e que nos últimos anos têm um aumento do desenvolvimento de experiências na Educação Básica; em relação ao desenvolvimento de jogos, no trabalho de [Boucinha et al. 2017] é apresentada uma prática pedagógica desenvolvida por meio de um curso ofertado como atividade extraclasse, tendo como público alvo estudantes do Ensino Fundamental. Para a atividade prática, o trabalho apresentou uma metodologia para ensino de PC por meio de desenvolvimento de jogos utilizando Scratch, bem como a avaliação de sua eficácia. Já [Pessoa et al. 2017] utilizaram um aplicativo com gamificação chamado T-mind para estimular o desenvolvimento de habilidades do PC. Segundo o estudo, os resultados apontaram que o uso do aplicativo pode estimular cinco habilidades: análise de dados, coleta de dados, decomposição, abstração e representação de dados, Já [Avila et al. 2017] apresentaram uma RSL sobre o ensino do PC por meio da Robótica no Ensino Básico. De acordo com o estudo, a temática do desenvolvimento de competências e habilidades usando a robótica como estratégia ainda é bastante recente, na qual diversas questões estão em aberto, principalmente metodológicas, no sentido de guiar a realização de intervenções que visem desenvolver habilidades do PC.

Outra estratégia que pode ser utilizada é o ensino do PC por meio de tecnologias de IoT. Segundo [Coetzee and Eksteen 2011], a IoT é um conceito relacionado à conexão de objetos entre eles e à internet, como sensores de qualidade de água e ar, sensores de tráfego de veículos e pessoas, câmeras de vídeo, dentre outros. Na IoT, esses objetos devem ser identificados, sua localização e estado conhecidos devem ser conectados à internet e estarem acessíveis por meio de uma rede interoperável. Podem, ainda, controlar e serem controlados remotamente. Atualmente, houve um significativo aumento da capacidade de processamento dos dispositivos de IoT. A evolução da tecnologia e o consequente 
VIII Congresso Brasileiro de Informática na Educação (CBIE 2019)

Anais do XXX Simpósio Brasileiro de Informática na Educação (SBIE 2019)

barateamento dos sensores e atuadores tornou a IoT mais aplicável a diferentes cenários, como cidades, casas, hospitais e hortas inteligentes; monitoramento de variáveis ambientais para alerta e prevenção de inundações e incêndios e na Indústria 4.0. Na Educação, essa grande quantidade de aplicabilidade abre oportunidades da utilização de IoT para o ensino do PC, visto que, por meio da IoT, o estudante é capaz de realizar a abstração, decomposição, analisar e encontrar padrões e desenvolver algoritmos para projetos que envolvam sensores e atuadores para resolver problemas reais.

Enquanto a robótica educacional tem como intuito o desenvolvimento de um componente, seja ele autônomo ou não, a IoT possui aspectos como comunicabilidade entre vários componentes, visualização, segurança e integridade dos dados coletados por sensores. Alguns trabalhos têm ainda por objetivo o uso de objetos de IoT e/ou ferramentas de IoT para o ensino de Computação e/ou outros conteúdos, por exemplo, em [Moreira and Baranauskas 2017] foi investigado o potencial da IoT como mediadora de processos de aprendizagem em ambiente educacional, segundo os autores "a tecnologia pode ser usada para instigar e inspirar a construção de narrativas, além de promover habilidades de expressão, formulação e sequenciação de ideias". Além disso, o uso de microcontroladores como Arduino para o ensino de programação não necessariamente pode ser considerado ensino de IoT e/ou ensino do PC por meio da IoT. Assim, é necessário compreender como a IoT vem sendo utilizada para o ensino do PC, com quais objetivos, metodologias, ferramentas e sobre quais conteúdos. Na seção a seguir, apresentamos a metodologia utilizada para a execução da RSL para responder a esses questionamentos.

\section{Metodologia da RSL}

A RSL apresentada neste trabalho seguiu a metodologia de [Brereton et al. 2007], cujo protocolo segue as etapas de: especificação das questões de pesquisa, definição dos termos de busca, escolha das bases, critérios de inclusão e de exclusão e síntese dos trabalhos selecionados. Por ser relevante obter trabalhos atuais e devido à evolução dos assuntos sobre PC e IoT, optou-se por escolher trabalhos publicados nos últimos cinco anos, com o objetivo de identificar, analisar e interpretar estudos publicados nos principais meios de divulgação acadêmicos, que apresentam como os conceitos de IoT podem ser utilizados no ensino do PC e para isso buscou-se responder às seguintes questões de pesquisa:

QP1: Quais são os principais objetivos dos estudos pesquisados?

QP2: Quais metodologias estão sendo utilizadas para o uso da IoT no ensino do PC?

QP3: Quais ferramentas estão sendo utilizadas para incorporar IoT no ensino do PC?

QP4: Quais conteúdos da área de Computação são abordados?

A QP1 visa mapear os objetivos dos estudos selecionados, tendo em vista que pode haver diversas abordagens sobre o mesmo conteúdo. Na QP2, pretende-se identificar as metodologias educacionais utilizadas e como são realizados os estudos em outros países. Na QP3 pretende-se identificar quais ferramentas tecnológicas estão sendo utilizadas no ensino do PC pelo conceito de IoT. E, na QP4, busca-se relacionar os conteúdos de Ciência da Computação que são ensinados.

A partir das questões de pesquisa, foi elaborada a string de busca: ("internet of things" OR "internet das coisas") AND (“computational thinking” OR "pensamento computacional”), aplicadas aos seguintes repositórios: ACM Digital Library, EI Conpendex, IEEE Xplore, ISI Web of Science, Science Direct, Scopus e Springer Link. 
VIII Congresso Brasileiro de Informática na Educação (CBIE 2019)

Anais do XXX Simpósio Brasileiro de Informática na Educação (SBIE 2019)

Os critérios de inclusão para seleção dos trabalhos foram: CI1: trabalhos publicados nos últimos cinco anos; CI2: trabalhos completos e, CI3: trabalhos cujo conteúdo está relacionado às questões da pesquisa. Já os critérios de exclusão foram: CE1: trabalhos que não tenham ligação com o ensino do PC por meio de IoT; CE2: trabalhos duplicados, sendo o mais recente escolhido para ser analisado; CE3: capítulos de livros e artigos curto; e CE4: trabalhos não disponíveis para download.

Seguindo o protocolo, a busca inicial resultou em 100 trabalhos. Após análise dos critérios de inclusão e exclusão foram selecionados para análise apenas 7 trabalhos, pois: (i) a grande maioria dos trabalhos não tiham ligação com o ensino do PC por meio de IoT, dessa forma, os trabalhos cujo objetivo era o uso de objetos e/ou ferramentas de IoT para o ensino de Computação ou de outros conteúdos também foram excluídos, tendo em vista que o foco do presente trabalho é o uso dos conceitos de IoT para o desenvolvimento do PC; (ii) alguns trabalhos ainda não estavam disponíveis para download e: (iii) a atualidade do tema pesquisado, pois os trabalhos selecionados foram publicados em 2018.

\section{Análise dos Trabalhos Selecionados}

Observamos que os trabalhos selecionados foram realizados na Alemanha, Estados Unidos, Grécia, Itália e Suécia, não sendo encontrados trabalhos na América Latina. Conforme análise, são realizados experimentos em todos os grupos educacionais - ensino fundamental, médio, superior e na formação geral. Apesar da pequena quantidade de trabalhos, podemos observar que as pesquisas não estão se limitando apenas a um grupo educacional. Apresentamos a seguir a análise realizada à luz de cada questão de pesquisa.

\section{QP1: Quais são os principais objetivos dos estudos pesquisados?}

Identificamos três grandes principais objetivos e descrevemos os trabalhos em cada um deles:

\section{1 - Discussões, análises e relatos de experiência de IoT no ensino do PC}

Em [Magdalinou and Papadakis 2017] foram apresentados a estudantes de Ensino Médio o ensino de computação ubíqua, computação móvel e IoT. Os autores identificaram que houve melhora da capacidade cognitiva de resolução de problemas por parte dos estudantes, bem como motivação e interesse em escolher carreiras profissionais na área de tecnologia e engenharia. Segundo os autores, o maior desafio foi desenvolver formas de utilizar as características técnicas das tecnologias para levar ao aprendizado e não apenas ao acesso à informação aos estudantes.

No trabalho de [Viberg and Mavroudi 2018] eles analisam as respostas de dez especialistas acerca dos fatores de sucesso e dos desafios da computação ubíqua e IoT no desenvolvimento de habilidades e competências pelo estudante do século XXI. Segundo os especialistas, os estudantes são capazes de desenvolver, por meio do PC, habilidades sociais e éticas, incluindo comunicação, colaboração, pensamento crítico e criatividade e autorregulação, que, de acordo com [França and Tedesco 2015b], é a capacidade do estudante definir seus próprios objetivos, monitorar e avaliar a própria aprendizagem. $\mathrm{O}$ sucesso do uso dessas tecnologias em sala de aula depende do interesse e motivação dos alunos em utilizar os conhecimentos de tecnologias na solução de problemas do seu cotidiano. Outro fator de sucesso se deve à robustez, usabilidade e maturidade da tecnologia citada. O desafio elencado é referente à escalabilidade do uso da tecnologia em sala de 
VIII Congresso Brasileiro de Informática na Educação (CBIE 2019)

Anais do XXX Simpósio Brasileiro de Informática na Educação (SBIE 2019)

aula dados os custos.

Já em [Eriksson et al. 2018] os autores apresentam relatos de experiências e diferentes considerações sobre a implantação de laboratórios makers ou fablabs nas escolas primárias e secundárias da Suécia. Esses laboratórios têm por objetivo proporcionar o trabalho colaborativo e criativo utilizando conceitos de IoTs, eletrônica e programação, para desenvolver o PC nos estudantes. Outros objetivos são desenvolver motivação e interesse em ciência e tecnologia; desenvolver a capacidade dos alunos de se relacionarem com a mídia e a informação de maneira mais crítica e responsável e; inspirar formas criativas de resolver problemas. Os autores apontaram que o desafio à implantação dos laboratórios nas escolas é desenvolver estratégias curriculares com sequência didática, materiais de ensino, métodos e atividades que possam ser facilmente integradas e flexíveis para suportar diferenças do contexto escolar, prazos e diferentes níveis de aprendizagem.

Em [Lensing and Friedhoff 2018] é apresentada uma experiência de um currículo adaptável a diferentes públicos como crianças, estudantes do ensino superior, pesquisadores e empresários, para ser desenvolvido em laboratórios makers ou fablabs. Utilizam programação e conceitos de IoT com o intuito de desenvolver habilidades e competências em estudantes de engenharia para que estejam capacitados à Indústria 4.0. As habilidades desenvolvidas foram criatividade, resolução de novos problemas e PC, uma vez que os fablabs são ambientes no qual a aprendizagem é autorregulada, o conhecimento não necessariamente necessita de uma hierarquia e seu foco é na solução de um problema real, além da cooperação e troca de conhecimento em contextos interdisciplinares.

\section{2 - Uso de IoT como mediadora no processo de ensino e aprendizagem}

No trabalho de [Bonani et al. 2017] eles abordam objetos interativos baseados em IoT para o ensino do PC por meio de conceitos de grafos para crianças. Os problemas são modelados com grafos para entender suas propriedades e desenvolver algoritmos sobre eles. Ainda segundo o estudo, os artefatos tangíveis para o ensino do PC promovem a interação entre a abstração e o concretude, estimulando a aprendizagem.

Em [Kelly et al. 2018] fazem um estudo utilizando o ambiente de programação visual chamado BlockyTalk. O foco é ensinar os conceitos de redes de computadores e programação distribuída, no qual os estudantes podem desenvolver diferentes arquiteturas e várias abordagens de colaboração, como a programação em pares. Os conceitos de redes de computadores podem ser considerados como uma habilidade técnica e avançada. No entanto, um estudo de caso utilizando o BlockyTalk como ferramenta de programação mostrou que mesmo programadores iniciantes são capazes de construir sistemas de rede, o que sugere que trabalhar com redes é muito parecido com outros tópicos da Ciência da Computação, como algoritmos.

Já [De Luca et al. 2018] introduzem uma ferramenta de programação visual, denominada VIPLE (Visual IoT/Robotics Programming Language Environment), baseada na linguagem Microsoft VPL, que implementa conceitos de PC e é utilizada para programar plataformas livres de robótica e IoT, como Lego EV3, Arduino, Raspberry Pi, dentre outras. A ferramenta foi utilizada inicialmente em programas de verão para estudantes do Ensino Fundamental e Médio, que receberam uma visão geral de introdução à programação usando o robô Lego Ev3 e VIPLE para programação do robô. Os autores citam que houve uma diferença significativa com os programas de verão realizados, nos 
VIII Congresso Brasileiro de Informática na Educação (CBIE 2019)

Anais do XXX Simpósio Brasileiro de Informática na Educação (SBIE 2019)

quais eram usadas linguagens de programação textuais, com grande esforço na sintaxe, tal que com VIPLE foi possível focar mais no PC. Apesar da ferramenta suportar opções para trabalhar com IoT, o relato de experiencia foi focado na robótica educacional.

\section{3 - Hardware para o ensino da IoT para o desenvolvimento do PC}

Foram utilizados hardware baseados em microcontroladores de placa única como Arduíno e Raspberry Pi, além de uma variedade de sensores e atuadores. Alguns trabalhos não citaram as ferramentas para programação das placas únicas, porém, pela leitura podemos inferir que foi utilizada a plataforma de desenvolvimento Arduíno IDE.

\section{QP2: Quais metodologias estão sendo utilizadas para o ensino do PC por meio do conceito de IoT?}

Nenhum dos trabalhos apresentou uma formalização de metodologias utilizadas para o ensino do PC por meio do conceito de IoT.

\section{QP3: Quais ferramentas estão sendo utilizadas para incorporar IoT no en- sino do PC?}

De acordo com o objetivo 3 da QP1, [Bonani et al. 2017] utilizam objetos interativos baseados em IoT; [Kelly et al. 2018] propõem o BlockyTalk e; [De Luca et al. 2018] propõem o VIPLE.

\section{QP4: Quais são os conteúdos da computação abordados?}

Com relação ao conteúdo, em [Magdalinou and Papadakis 2017] são ensinados conceitos sobre microcontroladores de placa única, eletrônica e programação básica utilizando o conceito de variáveis e estrutura condicional. [Lensing and Friedhoff 2018] utilizam conceitos de eletrônica básica, sensores e atuadores, algoritmos, programação básica e redes de computadores. [Kelly et al. 2018] trabalham com design de interação, objetivando a modelagem e o desenvolvimento de projetos. Logo, observa-se que eletrônica básica, sensores, atuadores, algoritmo, programação básica e redes de computadores são conteúdos essenciais em um cenário educacional voltado para o ensino do PC por meio do conceito de IoT. Observamos também que existem variações na carga horária entre os cenários educacionais. [Magdalinou and Papadakis 2017] utilizaram 8 horas, divididas em duas aulas de 4 horas e o projeto final. Já no cenário proposto por [Kelly et al. 2018], foram utilizadas 11 horas divididas em aulas de 1 hora e o projeto final.

\section{Análise do Cenário Atual e Perspectivas}

Por meio das questões de pesquisas analisamos que o ensino do PC por meio dos conceitos de IoT é um assunto emergente, porém com um vasto campo por explorar, como: Qual o conteúdo de computação e IoT que deve ser ensinado? Qual a carga horária da aula ou oficina ou minicurso? Que metodologia deverá ser aplicada? Como descrevemos por meio da QP2 os trabalhos carecem de uma formalização de metodologias utilizadas para o ensino do PC por meio do conceito de IoT.

Outro fator importante a ser analisado é como avaliar se o ensino do PC por meio da IoT, desenvolve habilidades e competências nos estudantes? De acordo com [Lensing and Friedhoff 2018], avaliar e mensurar a criatividade e o desenvolvimento de habilidades e competências adquiridas em um cenário educacional inovador é muito subjetivo. Assim, a avaliação qualitativa é mais indicada nesses casos. Nesse sentido, 
VIII Congresso Brasileiro de Informática na Educação (CBIE 2019)

Anais do XXX Simpósio Brasileiro de Informática na Educação (SBIE 2019)

em [De Luca et al. 2018] e [Bonani et al. 2017] a avaliação foi qualitativa, realizada por meio de observação e entrevista. Em [Kelly et al. 2018] a abordagem qualitativa foi realizada por meio de gravação de áudios e vídeos com consentimento dos participantes, para análise posterior. Em [Magdalinou and Papadakis 2017] a avaliação foi realizada por meio de dois questionários, um pré-teste e um pós-teste. Os questionários rastreavam as atitudes dos estudantes com relação à tecnologia e a sua capacidade de resolver problemas, o conhecimento dos termos e princípios relacionados à operação de tecnologia de informação (TI), bem como o interesse e autoconfiança dos estudantes em utilizar a tecnologia e sua intenção de seguir carreira na área de Exatas.

Mesmo considerando os desafios citados, a utilização da IoT no ensino do PC contribui para o desenvolvimento de habilidades e competências necessárias aos estudantes do século XXI: autorregulação, comunicação, colaboração, pensamento crítico e criatividade. Por meio dos conceitos de IoT é possível motivar e despertar o interesse dos estudantes, pois o aprendizado do PC pode ser mais significativo quando ultrapassa a esfera teórica e sua representação apenas no computador. Por exemplo, o estudante pode desenvolver um projeto que emita um sinal sonoro com base na leitura de um sensor de umidade. Logo, ele é capaz de ver, ouvir e tocar aquilo que ele programou, provendo assim a interação entre a abstração do problema e a concretude da solução, estimulando assim o desenvolvimento de novos conhecimentos, habilidades e competências.

Além disso, é imprescindível que quando o assunto é IoT, deva-se discutir que os dados são coletados pelos mais diversos objetos conectados à rede de computadores, que podem ser câmeras de segurança, monitores cardíacos, sensores de temperatura e umidade, dentre outros, o que traz reflexões éticas importantes nesse cenário: Qual o nível de acesso a esses dados? Que dados podem ficar disponíveis a todos? Qual o papel do cidadão neste cenário?. Essas perguntas vão ao encontro da Lei de Acesso à Informação ${ }^{1}$ e da Lei Geral de Proteção de Dados Pessoais², e nenhuma dessas questões foram abordadas em nenhum dos estudos analisados neste trabalho. No entanto, a conscientização dos estudantes nesse contexto é de extrema importância. Se faz necessário então o desenvolvimento de estratégias curriculares com sequências didáticas, materiais de ensino, métodos e atividades que possam ser facilmente integradas e flexíveis para a realidade da Educação brasileira, bem como definir metodologias para avaliar quais habilidades e competências são desenvolvidas com o ensino do PC por meio dos conceitos de IoT.

\section{Considerações Finais}

Com o evolução ao acesso às tecnologias digitais, é imperativo que a sociedade compreenda que a Computação é uma ciência capaz, não apenas de construir artefatos diversos, mas também, capaz de auxiliar no desenvolvimento de habilidades de competências necessárias aos estudantes do século XXI. Pesquisas mostram que é importante que o estudante desenvolva as habilidades do PC, além de habilidades de comunicação, colaboração, pensamento crítico, criatividade, resolução de novos problemas e cooperação, sendo que estas podem ser alavancadas por práticas educativas tendo o desenvolvimento do PC como viés metodológico, e que podem ainda, motivar e despertar interesse nas Ciências Exatas.

\footnotetext{
${ }^{1}$ Lei Federal N. 12.527, de 18 de novembro 2011, disponível em http: / / www.planalto.gov . br/ccivil_03/_ato2011-2014/2011/lei/112527.htm.

${ }^{2}$ Lei Federal N. 13.709, de 14 de agosto de 2018, disponível em http://www.planalto.gov. br/ccivil_03/_Ato2015-2018/2018/Lei/L13709.htm.
} 
Para desenvolvimento dessas habilidades por meio do PC são utilizadas e descritas na literatura o uso de ferramentas como computação desplugada, algoritmos, introdução à programação - por vezes com foco na construção de jogos e robótica, como iniciativas mais comuns. Nesse contexto, a revisão sistemática realizada investigou o estado atual e perspectivas de pesquisas a partir de artigos onde discussões, análise e relatos de experiência de IoT no ensino do PC estivessem presentes, tendo uso de IoT como mediadora no processo de ensino e aprendizagem de Computação e hardware para o ensino de IoT para o desenvolvimento do PC.

Os trabalhos analisados mostraram que é possível utilizar a IoT no ensino do PC, e o aprendizado pode ter mais significado aos alunos a partir de problemas situados em seus contextos. Porém, pela atualidade, o tema possui várias questões em aberto, sobretudo metodológicas tanto na intervenção quanto na avaliação das habilidades de PC desenvolvidas, além de questões éticas e de privacidade de dados que também precisam ser discutidas nesse cenário, com vistas a desenvolver nos estudantes a criticidade e a capacidade de resolução de problemas. Como trabalhos futuros pretendemos elaborar um estudo para o cenário educacional brasileiro por meio de uma intervenção educacional com estudantes do ensino médio, utilizando uma sequência didática com materiais de ensino, métodos e atividades com conteúdo voltado para o desenvolvimento de habilidades do PC por meio da IoT e questões éticas de segurança e privacidade dos dados. Para assim colaborar com o desenvolvimento dessa área de pesquisa.

\section{Referências}

Avila, C., Cavalheiro, S., Bordini, A., and Marques, M. (2017). O pensamento computacional por meio da robótica no ensino básico - uma revisão sistemática. In Anais do XXVIII Simpósio Brasileiro de Informática na Educação, volume 28, pages 82-91.

Barcelos, T., Muñoz, R., Acevedo, R. V., and Silveira, I. F. (2015). Relações entre o pensamento computacional e a matemática: uma revisão sistemática da literatura. In Anais dos Workshops do IV Congresso Brasileiro de Informática na Educação, volume 4, pages 1369-1378.

Blikstein, P. (2008). O pensamento computacional e a reinvenção do computador na educação. Education \& Courses.

Bonani, A., Del Fatto, V., Dodero, G., Gennari, R., and Raimato, G. (2017). Participatory design of tangibles for graphs: A small-scale field study with children. In Conference on Smart Learning Ecosystems and Regional Development, pages 161-168. Springer.

Boucinha, R. M., Brackmann, C. P., Barone, D. A. C., and Casali, A. (2017). Construção do pensamento computacional através do desenvolvimento de games. RENOTE, 15(1).

Brereton, P., Kitchenham, B. A., Budgen, D., Turner, M., and Khalil, M. (2007). Lessons from applying the systematic literature review process within the software engineering domain. Journal of systems and software, 80(4):571-583.

Coetzee, L. and Eksteen, J. (2011). Internet of things-promise for the future? an introduction. Proceedings of the 2011 IST-Africa Conferenc.

De Luca, G., Li, Z., Mian, S., and Chen, Y. (2018). Visual programming language environment for different iot and robotics platforms in computer science education. CAAI Transactions on Intelligence Technology, 3(2):119-130. 
VIII Congresso Brasileiro de Informática na Educação (CBIE 2019)

Anais do XXX Simpósio Brasileiro de Informática na Educação (SBIE 2019)

Eriksson, E., Heath, C., Ljungstrand, P., and Parnes, P. (2018). Makerspace in school-considerations from a large-scale national testbed. International journal of child-computer interaction, 16:9-15.

França, R. and Tedesco, P. (2015a). Desafios e oportunidades ao ensino do pensamento computacional na educação básica no brasil. In Anais dos Workshops do Congresso Brasileiro de Informática na Educação - CBIE, volume 4, pages 1464-1473.

França, R. S. and Tedesco, P. C. d. A. R. (2015b). Um modelo colaborativo para a aprendizagem do pensamento computacional aliado à autorregulação. In Anais dos Workshops do Congresso Brasileiro de Informática na Educação - CBIE, volume 25, pages 85-94.

Kaminski, M. and Boscarioli, C. (2018). Production of scratch learning objects by elementary school students. In Conferência Latino-americana de Tecnologias de Aprendizagem.

Kelly, A., Finch, L., Bolles, M., and Shapiro, R. B. (2018). Blockytalky: New programmable tools to enable students' learning networks. International Journal of ChildComputer Interaction.

Lensing, K. and Friedhoff, J. (2018). Designing a curriculum for the internet-of-thingslaboratory to foster creativity and a maker mindset within varying target groups. Procedia Manufacturing, 23:231-236.

Magdalinou, K. and Papadakis, S. (2017). The use of educational scenarios using stateof-the-art it technologies such as ubiquitous computing, mobile computing and the internet of things as an incentive to choose a scientific career. In Interactive Mobile Communication, Technologies and Learning, pages 915-923. Springer.

Moreira, E. and Baranauskas, M. C. (2017). Alice das coisas: entendendo a comunicação entre objetos na construção de ambientes de aprendizagem. In Simpósio Brasileiro de Informática na Educação, volume 28, page 1017.

Ortiz, J. and Pereira, R. (2018). Um mapeamento sistemático sobre as iniciativas para promover o pensamento computacional. In Simpósio Brasileiro de Informática na Educação, volume 29, pages 1093-1102.

Papert, S. (1980). Mindstorms: Children, computers, and powerful ideas. Basic Books.

Pessoa, F. I. R., Araujo, A. L. S. O., Andrade, W., and Guerrero, D. (2017). T-mind: um aplicativo gamificado para estímulo ao desenvolvimento de habilidades do pensamento computacional. In Simpósio Brasileiro de Informática na Educação, volume 28, pages $645-654$.

Viberg, O. and Mavroudi, A. (2018). The role of ubiquitous computing and the internet of things for developing 21 st century skills among learners: Experts' views. In European Conference on Technology Enhanced Learning, pages 640-643. Springer.

Werlich, C., Crema, C., Kemczinski, A., and Gasparini, I. (2018). Pensamento computacional no ensino fundamental i: um estudo de caso utilizando computação desplugada. In Anais dos Workshops do Congresso Brasileiro de Informática na Educação, volume 7, pages 719-728.

Wing, J. (2006). Computational thinking. Communications of the ACM, 49(3):33-35. 